\title{
A STUDY ON MAGNETO HYDRODYNAMICS JEFFERY-HAMEL FLOW WITH HEAT TRANSFER PROBLEM IN EYRING-POWELL FLUID USING DIFFERENTIAL TRANSFORM METHOD
}

\author{
Ramakanta Meher, N.D. Patel \\ Department of Mathematics, S.V National Institute of Technology, Surat, Gujarat, India \\ meher_ramakanta@yahoo.com,nirmaths@gmail.com
}

Received: 23 June 2019; Accepted: 17 October 2018

\begin{abstract}
In this paper, we study and analyse the variations of velocity profiles for different values of the Reynolds number, Eckert number, Prandtl number and Hartmann number in the Magneto Hydrodynamics Jeffery-Hamel flow with heat transfer in Eyring-Powell fluid in both divergent and convergent channels. The Differential Transform Method (DTM) is used to obtain an analytical solution of the Jeffery Hamel flow problem and to determine the velocity profiles of the fluid flow. Finally, the efficiency of DTM has been shown, and the results have been validated by comparing the obtained results with the numerical results (fourth order RK method) in both convergent and divergent channels.
\end{abstract}

MSC 2010: 65N99, 76099

Keywords: Differential Transform Method, Jeffery-Hamel flow, Eyring-Powell fluid, heat transfer

\section{Nomenclature}

$\begin{array}{ll}p & \text { Pressure } \\ k & \text { Conductivity of the thermal } \\ \rho & \text { Density } \\ T & \text { Temperature } \\ \operatorname{Re} & \text { Reynolds number }\end{array}$

\section{Introduction}

The study on fluid flow between two inclined planes is a most important problem in the field of fluid flow problems in many fields of Engineering and Sciences. In the mid of nineteen century, Jeffery [1] and Hamel [2] derived a mathematical formulation for the fluid flow between two unparalled walls and studied the behaviour of a velocity profile and the effect of an angle on fluid flow in both divergent 
and convergent channels. Due its major importance in the field industry applications, Powell-Earring introduced a new model in 1944-45, which is now known as the Eyring-Powell model. Many investigators have examined the Jeffery Hamel Flow from different points of view. Akulenko et al. [3] analysed JH flow in a convergent channel with different values of Reynolds numbers while Makinde and Mhone [4] and Esmaili et al. [5] analysed itthrough semi-numerical approach i.e. Hermite-Padé approximation and the Adomian Decomposition Method. Rivkind et al. [6] computed it numerically with a finite number of outlets to infinity. Egashira, Fujikawa et al. [7] studied the Microscopic and low Reynolds number flows between two intersecting permeable walls while Rana, Shukla et al. [8] analytically predicted the multiple solutions for MHD Jeffery-Hamel flow and heat transfer by utilizing the KKL nanofluid modelSimilarly, many investigators have taken a keen involvement in various analytical and numerical methods like the Adomain Decomposition Method, the Perturbation Method, the Homotopy Analysis Method, the Modified Sumudu Transform Method and the Traveling Wave Transformation Method in the study of fluid flow problems and other fluid mechanics problems that are inherently non-linear. In fact, in most of the cases such problems do not possess numerical solutions so an analytical solution also plays an important role in the study of nonlinear differential equations. Muhmmad [9] used DTM and analysed the effect of the magnetic field between two parallel walls for unsteady double phases of nano-fluid flow and heat transfer. Zhou and Pukchov [10] and Hossein et al. [11] applied DTM and solved the electric circuit analysis and the non-linear Gas Dynamics and Klein-Gordon equations arising in fluid flow problems. Patil and Khambayat [12] used DTM for a linear differential equation while Chen and Ho [13] and Ayaz [14] extended the method for a non-linear system of an ordinary and partial differential equation. At a later period of time, Shahmorad et al. [15] and Farshid [16, 17] extended the applications of DTM to a fractional order integro-differential equation with non-vocal boundary conditions and also to a three-dimensional fuzzy partial differential equations. Patel and Meher [18-20] used DTM for the solution of Kolmogrove-Petrovskii-Piskunov equation and the porous medium equation and validated the obtained results by comparing with the exact solution. Rana, Shukla et al. [21] used the Homotopy analysis method for predicting multiple solutions in the channel flow with stability analysis.

In this paper, the MHD Jeffery-Hamel fluid flow in Eyring-Powell fluid between two non-parallel plates with heat transfer is considered to analyse the effects of the Eckert number, Prandtl number, Reynolds number and the Hartmann number on velocity profiles of fluid flow. The Differential Transform Method is used to study the variation of velocity profiles during MHD Jeffery Hamel flow for different values of Eckert number, Prandtl number, Reynolds number and the Hartmann number in both divergent and convergent channels and also checked the accuracy and the validity of the obtained results by comparing the obtained results with the results obtained by numerical results (fourth order RK method). 


\section{Mathematical formulation and solution of the problem}

For Eyring fluid, the extra - stress tensor is given as,

$$
\tau=\left(\mu_{s}+\frac{1}{\psi v} \sinh ^{-1}\left(\frac{v}{t}\right)\right) K
$$

where $v=\sqrt{\frac{1}{2} \operatorname{tr} K^{2}}$ and $K=\nabla V+(\nabla V)^{t}$ is kinematical tensor, $\psi$ and $t$ are the characteristics of the Eyring-Powell model and time, $t$ has the dimension of

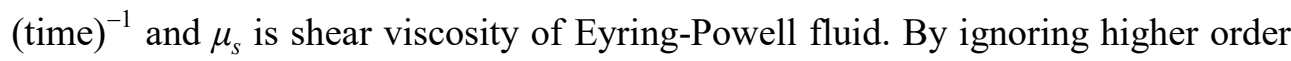
terms in expansion of the sine hyperbolic function of eq. (1), we get

$$
\tau=\mu_{s} K+\frac{1}{\psi t} K+\frac{v^{2} K}{6 \psi t^{3}}
$$

The shears of the Eyring-Powell Model in polar coordinates are defined as follows:

$$
\begin{gathered}
\tau_{l l}=\left(\mu_{s}+\frac{1}{\psi t}\right) \frac{2 \partial f}{\partial l}+\frac{v^{2}}{6 \psi t^{3}} \frac{2 \partial f}{\partial r} \\
\tau_{l \alpha}=\left(\mu_{s}+\frac{1}{\psi t}\right)\left(\frac{1 \partial f}{l \partial \alpha}+\frac{\partial g}{\partial l}-\frac{g}{l}\right)+\frac{v^{2}}{6 \psi t^{3}}\left(\frac{1 \partial f}{l \partial \alpha}+\frac{\partial g}{\partial l}-\frac{g}{l}\right) \\
\tau_{\alpha \alpha}=2\left(\mu_{s}+\frac{1}{\psi t}\right)\left(\frac{1 \partial g}{l \partial \alpha}+\frac{f}{l}\right)+\frac{v^{2}}{6 \psi t^{3}}\left(\frac{1 \partial g}{l \partial \alpha}+\frac{f}{l}\right) \\
v^{2}=2\left(\frac{\partial f}{\partial l}\right)^{2}+\left(\frac{\partial g}{\partial l}+\frac{1 \partial f}{l \partial \alpha}-\frac{g}{l}\right)^{2}+2\left(\frac{1}{l} \frac{\partial g}{\partial \alpha}+\frac{f}{l}\right)^{2}
\end{gathered}
$$

The scalar momentum equations in $\mathrm{r}$ and $\alpha$ directions are defined as follows:

$$
\begin{gathered}
\rho\left(\frac{\partial f}{\partial t}+f \frac{\partial f}{\partial l}+\frac{g \partial f}{l \partial \alpha}-\frac{g^{2}}{l}\right)=-\frac{\partial p}{\partial l}+\frac{1}{l} \frac{\partial\left(r \tau_{l l}\right)}{\partial l}+\frac{1}{l} \frac{\partial\left(\tau_{l \alpha}\right)}{\partial l}-\frac{\tau_{\alpha \alpha}}{l} \\
\rho\left(\frac{\partial g}{\partial t}+f \frac{\partial g}{\partial l}+\frac{g \partial g}{l \partial \alpha}+\frac{f g}{l}\right)=-\frac{1}{l} \frac{\partial p}{\partial l}+\frac{\partial\left(\tau_{l \alpha}\right)}{\partial l}+\frac{1}{l} \frac{\partial\left(\tau_{\alpha \alpha}\right)}{\partial \alpha}+2 \frac{\tau_{l \alpha}}{l}
\end{gathered}
$$

where $p$ is the pressure term of the Eyring-Powell model.

Consider an Eyring-Powell fluid flow between two inclined planes having angles $2 \alpha$ and $E_{0}$ electromagnetic induction as shown in Figure 1. The fluid flow can be considered as a convergent/divergent channel according to the angle $\alpha$ is negative/positive respectively. For fluid flow, it is assumed that there is no move- 
ment of flow in a vertical direction i.e. flow is purely radial in motion and $M$ is the external magnetic field action on flow. In addition, there is a constant temperature $T_{0}$ near the plates. With all the above conditions, the continuity, momentum and heat transfer equations can be defined in polar coordinates as:

$$
\begin{gathered}
\frac{1}{l} \frac{\partial}{\partial l}(l f)=0 \\
\rho f \frac{\partial f}{\partial l}=-\frac{\partial p}{\partial l}+\frac{\partial \tau_{l l}}{\partial l}+\frac{1}{l} \frac{\partial \tau_{l \alpha}}{\partial l}+\frac{\tau_{l l}-\tau_{\alpha \alpha}}{l}-E_{0} M^{2} \frac{f}{l^{2}} \\
0=-\frac{1}{l} \frac{\partial p}{\partial \alpha}+\frac{\partial \tau_{l \alpha}}{\partial l}+\frac{1}{l} \frac{\partial \tau_{\alpha \alpha}}{\partial \alpha}+2 \frac{\tau_{l \alpha}}{l} \\
\rho C_{p} f \frac{\partial T}{\partial l}=k\left(\frac{\partial^{2} T}{\partial l^{2}}+\frac{1}{l} \frac{\partial T}{\partial l}+\frac{1}{l^{2}} \frac{\partial^{2} T}{\partial \alpha^{2}}\right)+\frac{2 \partial f}{\partial l}+\frac{1}{l} \frac{\partial \tau_{l \alpha}}{\partial l} \tau_{l l}+\frac{2 f}{l} \tau_{\alpha \alpha}+\frac{2}{l} \frac{\partial f}{\partial \alpha} \tau_{l \alpha}
\end{gathered}
$$

with boundary conditions: $\frac{\partial f(l, \alpha)}{\partial \alpha}=0, \frac{\partial T}{\partial \alpha}=0, f(l, \alpha)=F_{\max }$ at the midline of the flow, $f(l, \alpha)=0$ and $T=T_{0}$ at the walls of the channel.

Where $C_{p}, E_{0}$ and $k$ are the specific heat, electrical conductivity and thermal conductivity respectively and the velocity of the flow at the centreline (i.e $\alpha=0$ ) is $F_{\text {max }}$.

From equation (9), $\quad u(\alpha)=l f(l, \alpha)$

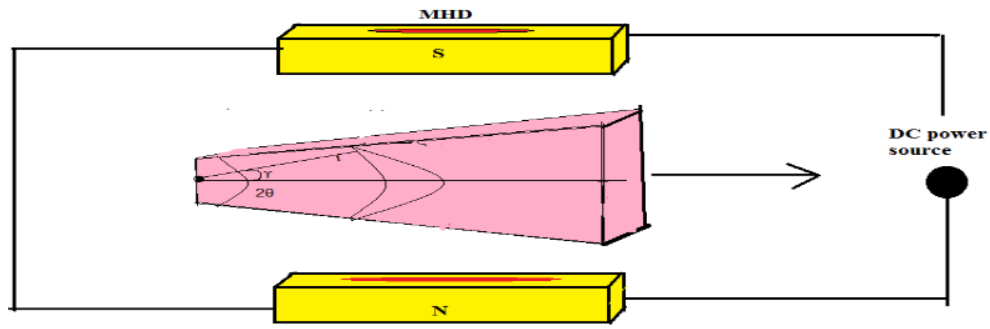

Fig. 1. Schematics diagram of the Jeffery Hamel Flow

Defining: $\quad u(\eta)=\frac{u(\theta)}{u_{\max }}, v(\eta)=\frac{T}{T_{0}}$ and $\eta=\frac{\alpha}{\theta}$

By eliminating the pressure term from eqs. (10)-(12) and using the above transformation we have:

$$
\begin{aligned}
\left(1+E_{p}\right)\left(u^{\prime \prime \prime}+4 \theta^{2} u^{\prime}\right) & +2 \operatorname{Re} \theta u u^{\prime}-\operatorname{Ha} \theta^{2} u^{\prime}-\frac{3 E_{p} \zeta}{\theta^{2}}\left(\frac{1}{2} u^{\prime 2} u^{\prime \prime \prime}+u^{\prime} u^{\prime \prime}\right) \\
& -\zeta E_{p}\left(72 u^{2} u^{\prime}+2 u^{\prime^{3}}+32 u u^{\prime} u^{\prime \prime}+2 u^{2} u^{\prime \prime \prime}\right)=0
\end{aligned}
$$




$$
v^{\prime \prime}+\operatorname{Pr} E_{c}\left(2\left(1+E_{p}\right)\left(u^{\prime 2}+4 \theta^{2} u^{2}\right)-8 E_{p} \zeta\left(u^{\prime 2}+2 \theta^{2} u^{2}\right) u^{2}-\frac{E_{p} \zeta}{\theta^{2}} u^{\prime 4}\right)=0
$$

Subject to the boundary conditions

$$
u(0)=1, u^{\prime}(0)=0, u(1)=0, v^{\prime}(0)=0 \text { and } v(1)=0
$$

where $\zeta=\frac{F_{\max }^{2}}{3 l^{2} t^{2}}$ is the local non-Newtonian parameter, $E_{p}=\frac{1}{\mu \beta t}$ is the dimensionless Eyring-Powell parameter, $\operatorname{Re}=\frac{\theta u_{\max }}{v}$ is the Reynolds number, $\mathrm{Ha}=\frac{E_{0} M^{2}}{\mu}$ is the Hartmann number, $\operatorname{Pr}=\frac{\mu C_{p}}{k}$ is the Prandtl number and $E_{c}=\frac{F_{\max }^{2}}{T_{0} C_{p}}$.

\section{Solution of the MHD Jeffery-Hamel flow with heat transfer problem using the Differential Transform Method}

The differential transform of function $e(\eta)$ can be defined as follows:

$$
E(k)=\frac{1}{k !}\left[\frac{d^{k} e(\eta)}{d \eta^{k}}\right]_{\eta=0}
$$

where $e(\eta)$ is original function and $E(k)$ is the transformed function. The uppercase and lowercase letters represent the transformed and original function respectively. The inverse differential transform of $E(k)$ is defined as:

$$
e(\eta)=\sum_{0}^{\infty} E(k) \eta^{k}
$$

Using equation (8) in (9), it gives, $e(\eta)=\sum_{0}^{\infty}\left[\frac{d^{k} e(\eta)}{d \eta^{k}}\right]_{\eta=0} \frac{\eta^{k}}{k !}$

By applying the fundamental operations of the differential transform method to equation (16)-(19), it obtains,

$$
\begin{aligned}
& \left(1+e_{p}\right)(k+1)(k+2)(k+3) U(k+3)+4 \theta^{2}\left(1+e_{p}\right)(k+1) U(k+1)+2 \operatorname{Re} \theta \sum_{k_{1}=0}^{k}\left(k_{1}+1\right) U\left(k_{1}+1\right) U\left(k-k_{1}\right)-\operatorname{Ha} \theta^{2}(k+1) U(k+1)- \\
& \frac{3 e_{p} \sigma}{2 \theta^{2}} \sum_{k_{2}=0}^{k} \sum_{k_{1}=0}^{k_{2}}\left(k_{1}+1\right)\left(k_{1}+2\right)\left(k_{1}+3\right)\left(k_{2}-k_{1}+1\right)\left(k-k_{2}+1\right) U\left(k_{1}+3\right) U\left(k_{2}-k_{1}+1\right) U\left(k-k_{2}+1\right) \\
& -\frac{3 e_{p} \sigma}{2 \theta^{2}} \sum_{k_{2}=0}^{k} \sum_{k_{1}=0}^{k_{2}}\left(k_{1}+1\right)\left(k_{1}+2\right)\left(k_{2}-k_{1}+1\right)\left(k_{2}-k_{1}+2\right)\left(k-k_{2}+1\right) U\left(k_{2}+2\right) U\left(k_{2}-k_{1}+2\right) U\left(k-k_{2}+1\right) \\
& -72 e_{p} \sigma \sum_{k_{2}=0}^{k} \sum_{k_{1}=0}^{k_{2}}\left(k_{1}+1\right) U\left(k_{1}+1\right) U\left(k_{2}-k_{1}\right) U\left(k-k_{2}\right)-2 e_{p} \sigma \sum_{k_{2}=0}^{k} \sum_{k_{1}=0}^{k_{2}}\left(k_{1}+1\right)\left(k_{2}-k_{1}+1\right)\left(k-k_{2}+1\right) U\left(k_{1}+1\right) U\left(k_{2}-k_{1}+1\right) U\left(k-k_{2}+1\right) \\
& -32 e_{p} \sigma \sum_{k_{2}=0}^{k} \sum_{k_{1}=0}^{k_{2}}\left(k_{1}+1\right)\left(k_{2}-k_{1}+1\right) U\left(k_{1}+1\right) U\left(k_{2}-k_{1}+1\right) U\left(k-k_{2}\right) \\
& -26 e_{p} \sigma \sum_{k_{2}=0}^{k} \sum_{k_{1}=0}^{k_{2}}\left(k_{1}+1\right)\left(k_{1}+2\right)\left(k_{1}+3\right) U\left(k_{1}+3\right) U\left(k_{2}-k_{1}\right) U\left(k-k_{2}\right)=0
\end{aligned}
$$


and

$(k+1)(k+2) V(k+2)+2 \operatorname{Pr} E_{c}\left(1+e_{p}\right) \sum_{k_{1}=0}^{k}\left(k_{1}+1\right)\left(k-k_{1}+1\right) U\left(k_{1}+1\right) U\left(k-k_{1}+1\right)+8 \operatorname{Pr} E_{c}\left(1+e_{p}\right) \theta^{2} \sum_{k_{1}=0}^{k} U\left(k_{1}\right) U\left(k-k_{1}\right)$

$-8 \operatorname{Pr} E_{c} e_{p} \zeta \sum_{k_{3}=0}^{k} \sum_{k_{2}=0}^{k_{3}} \sum_{k_{1}=0}^{k_{2}}\left(k_{1}+1\right)\left(k_{2}-k_{1}+1\right) U\left(k_{1}+1\right) U\left(k_{2}-k_{1}+1\right) U\left(k_{3}-k_{2}\right) U\left(k-k_{3}\right)$

$-16 \operatorname{Pr} E_{c} e_{p} \zeta \theta^{2} \sum_{k_{3}=0}^{k} \sum_{k_{2}=0}^{k_{3}} \sum_{k_{1}=0}^{k_{2}} U\left(k_{1}\right) U\left(k_{2}-k_{1}\right) U\left(k_{3}-k_{2}\right) U\left(k-k_{3}\right)$

$-\frac{\operatorname{Pr} E_{c} e_{p} \zeta}{\theta^{2}} \sum_{k_{3}=0}^{k} \sum_{k_{2}=0}^{k_{3}} \sum_{k_{1}=0}^{k_{2}}\left(k_{1}+1\right)\left(k_{2}-k_{1}+1\right)\left(k_{3}-k_{2}+1\right)\left(k-k_{3}+1\right) U\left(k_{1}+1\right) U\left(k_{2}-k_{1}+1\right) U\left(k_{3}-k_{2}+1\right) U\left(k-k_{3}+1\right)=0$

Upon solving the above with the conditions (17), it obtain,

$$
\begin{aligned}
& u(\eta)=1+c_{1} \eta^{2}+\left(-\frac{1}{6} \theta \operatorname{Re} c_{1}+\frac{1}{12}\left(1+e_{p}\right) \zeta \operatorname{Ha} \theta^{2} c_{1}\right) \eta^{4} \\
& +\left(-\frac{\theta \operatorname{Re}\left(1+e_{p}\right) \zeta\left(2 c_{1}^{2}-\frac{2}{3} \theta \operatorname{Re} c_{1}+\frac{1}{3} \operatorname{Ha} \theta^{2} c_{1}\right)}{60}+\frac{1}{30} \operatorname{Ha} \theta^{2}\left(1+e_{p}\right) \zeta\left(-\frac{1}{6} \theta \operatorname{Re} c_{1}+\frac{1}{12} \operatorname{Ha} \theta^{2} c_{1}\right)\right) \eta^{6}+\ldots
\end{aligned}
$$

and

$$
\begin{aligned}
v(\eta) & =c_{2}+\left(-2 \operatorname{Pr} E_{c}\left(1+e_{p}\right) \xi \theta^{2} c_{2}-1 / 2\left(4 \theta^{2} \operatorname{Pr} E_{c}\left(1+e_{p}\right) \xi\right)\right) \eta^{2} \\
& +\left(-1 / 3 \theta^{2}\left(-2 c_{2}-\theta^{2} \operatorname{Pr} E_{c}\left(1+e_{p}\right) c_{2}-1 / 2\left(4 \theta^{2}+\operatorname{Pr} E_{c}\left(1+e_{p}\right) \xi\right)\right)-1 / 6 \theta^{2} \operatorname{Pr} E_{c}\left(1+e_{p}\right) \xi\right) \eta^{4}+\ldots
\end{aligned}
$$

In this particular case, if we take $\operatorname{Re}=20, \operatorname{Pr}=6.2, \theta=\frac{\pi}{180}$ and $\mathrm{Ha}=5, e_{p}=0.2$, $E_{c}=0.5$, we have,

$$
\begin{aligned}
u(\eta)= & 1-8.58205398866123 \eta^{2}+30.1810617207590 \eta^{4}-64.2383586265795 \eta^{6} \\
& +81.1002062211646 \eta^{8}-53.9770189687069 \eta^{10}+14.5162239625121 \eta^{12}+\ldots
\end{aligned}
$$

and

$$
\begin{aligned}
v(\eta)= & 1-14.9945780724458 \eta^{2}+75.8429204318254 \eta^{4}-205.127347727176 \eta^{6} \\
& +302.608310357512 \eta^{8}-225.744141352790 \eta^{10}+66.4148052619667 \eta^{12}+\ldots
\end{aligned}
$$

Equation (24) and (25) describe the velocity profiles of Jeffery Hamel flow between two non-parallel plates with heat transfer in Eyring-Powell fluid. 


\section{Convergence study of the solution}

Theorem: Let $\phi$ be an operator from a Hilbert space $H_{0}$ in to $H_{0}$ and let $E$ be an exact solution of Eq. (15) and (16). Then $\sum_{0}^{\infty} E(k) \gamma^{k}$ which is obtained by Eq. (19), converges to the exact solution, if there exists a $\psi, 0<\psi<1$ such that $\left\|E_{k+1}\right\| \leq \Psi\left\|E_{k}\right\|, \forall k \in \mathrm{N} \cup\{0\}$.

Proof: We have

$$
\begin{aligned}
S_{0}= & \\
S_{1}= & S_{0}+E_{1}=E_{1}, \\
S_{2}= & S_{1}+E_{2}=E_{1}+E_{2}, \\
& \vdots \\
S_{n}= & S_{n-1}+E_{n}=E_{1}+E_{2}+E_{3}+\ldots+E_{n}
\end{aligned}
$$

and we will show that $\left\{S_{n}\right\}_{n=0}^{\infty}$ is a Cauchy sequence in a Hilbert Space $H_{0}$. Now for

$$
\left\|S_{n+1}-S_{n}\right\|=\left\|E_{n+1}\right\| \leq \Psi\left\|E_{n}\right\| \leq \Psi^{2}\left\|E_{n-1}\right\| \leq \ldots \leq \Psi^{n+1}\left\|E_{0}\right\| \text { for every } n, m \in N, n \geq m
$$

we have,

$$
\begin{aligned}
\| S_{n}-S_{m} & \|=\|\left(S_{n}-S_{n-1}\right)+\left(S_{n-1}-S_{n-2}\right)+\cdots+\left(S_{m-2}-S_{m+1}\right)+\left(S_{m+1}-S_{m}\right) \| \\
& \leq\left\|S_{n}-S_{n-1}\right\|+\left\|S_{n-1}-S_{n-2}\right\|+\cdots+\left\|S_{m-2}-S_{m+1}\right\|+\left\|S_{m+1}-S_{m}\right\| \\
& \leq \Psi^{n}\left\|E_{0}\right\|+\Psi^{n-1}\left\|E_{0}\right\|+\Psi^{n-2}\left\|E_{0}\right\|+\cdots+\Psi^{m+2}\left\|E_{0}\right\|+\Psi^{m+1}\left\|E_{0}\right\| \\
& \leq\left(\Psi^{m+1}+\Psi^{m+2}+\cdots\right)\left\|E_{0}\right\|=\frac{\Psi^{m+1}}{1-\Psi}\left\|E_{0}\right\|
\end{aligned}
$$

Which implies $\lim _{n, m \rightarrow \infty}\left\|S_{n}-S_{m}\right\|=0$, i.e., $\left\{S_{n}\right\}_{0}^{\infty}$ is a Cauchy sequence in a Hilbert space $H$ and it converges to $S$ for $S \in H$.

Definition: For every $i \in N \cup\{0\}, \psi_{i}$ can be defined as

$$
\Psi_{i}=\left\{\begin{array}{c}
\frac{\left\|E_{i+1}\right\|}{\left\|E_{i}\right\|},\left\|E_{i}\right\| \neq 0 \\
0,\left\|E_{i}\right\|=0
\end{array}\right.
$$


Corollary: If $0 \leq \Psi_{i}<1, i=1,2,3, \ldots$, then $\sum_{i=0}^{\infty} E_{i}$ converges to the exact solution $E$. Now by Corollary, since $\Psi_{0}=\frac{\left\|E_{1}\right\|}{\left\|E_{0}\right\|}=0<1, \Psi_{1}=\frac{\left\|E_{2}\right\|}{\left\|E_{1}\right\|}=0<1, \Psi_{2}=\frac{\left\|E_{3}\right\|}{\left\|E_{2}\right\|}=0<1$, similarly, $\psi_{n}=0$ for all $\mathrm{n}$. Therefore $\sum_{0}^{\infty} E(k) \gamma^{k}$ is convergent.

\section{Results and discussion}

For the validity of the results, the obtained results have been compared with the available numerical results as shown in Table 1, and the error has been computed for $\operatorname{Re}=20, \operatorname{Pr}=6.2, \theta=\frac{\pi}{180}$ and $\mathrm{Ha}=5, E_{p}=0.2, E_{c}=0.5$, also from Figures $2-10$, it can be observed that there is a good agreement between the obtained and the available results.

Table I. Comparison between DTM (Differential Transform Method) and Numerical solution for $\operatorname{Re}=20, \operatorname{Pr}=6.2, \theta=\frac{\pi}{180}$ and $\mathrm{Ha}=5, e_{p}=0.2, E_{c}=0.5$

\begin{tabular}{|c|c|c|c|c|c|c|}
\hline & \multicolumn{4}{|c|}{$u(\eta)$} & & \\
\hline$\eta$ & DTM & Numerical & Error & DTM & Numerical & Error \\
\hline 0 & 1 & 1 & 0 & 1 & 1 & 0 \\
\hline 0.1 & 0.917134134 & 0.91701151 & 0.000122623 & 0.997652 & 0.998501 & 0.00085 \\
\hline 0.2 & 0.701098433 & 0.70097338 & 0.000125053 & 0.988524 & 0.988265 & 0.000259 \\
\hline 0.3 & 0.430261948 & 0.430132422 & 0.000129525 & 0.96939 & 0.969043 & 0.000347 \\
\hline 0.4 & 0.184119697 & 0.183976428 & 0.00014327 & 0.940561 & 0.940353 & 0.000207 \\
\hline 0.5 & 0.004708255 & 0.004545125 & 0.00016313 & 0.903678 & 0.903006 & 0.000672 \\
\hline 0.6 & -0.10778639 & -0.10796946 & 0.000183066 & 0.850736 & 0.850757 & $2.1 \mathrm{E}-05$ \\
\hline 0.7 & -0.164840653 & -0.16506377 & 0.00022312 & 0.757532 & 0.75726 & 0.000272 \\
\hline 0.8 & -0.161886148 & -0.16230921 & 0.000423066 & 0.601488 & 0.601144 & 0.000344 \\
\hline 0.9 & -0.098399565 & -0.09902268 & 0.00062312 & 0.385191 & 0.380578 & 0.004613 \\
\hline 1 & 0.00006031 & 0 & 0.00006031 & $4.51 \mathrm{E}-05$ & 0 & $4.51 \mathrm{E}-05$ \\
\hline
\end{tabular}

Figures 2-10 discusses the effect of various parameters like the angle, Hartmann number, Eckert number, Prandtl number and the Reynolds number on the velocity profile and heat profile of Jeffery Hamel Eyring-Powell fluid flow. From Figures 2 and 3, it can be observed that the velocity and the heat profile of Jeffrey Hamel Nano fluid flow decreases as the angle between two unparalleled walls increase 
keeping the value of the remaining parameters as fixed. Additionally, it shows that as the angle of the channel gets larger, both the profiles (Velocity and Heat) of Jeffery Hamel nano-fluid flow show the same increasing pattern, but the temperature profile of Jeffrey Hamel nano-fluid flow increases faster in a convergent channel as compared to the velocity profiles of Jeffrey Hamel nano-fluid flow in a divergent channel.

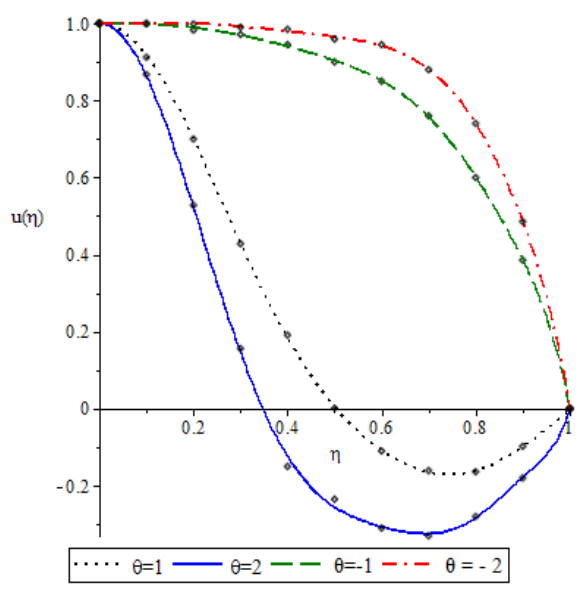

Fig. 2. The effect of angle on the velocity profile for both divergent $(\theta>0)$ and convergent $(\theta<0)$ channels

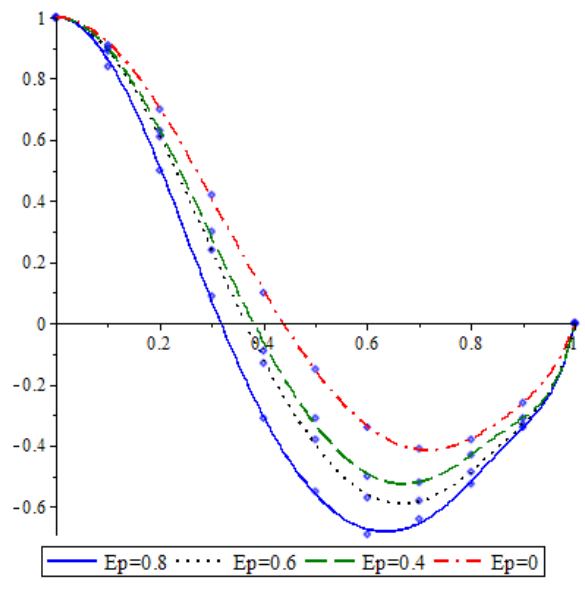

Fig. 4. The effect of $E_{p}$ on velocity profile for $\theta=2.5$ and $\mathrm{Re}=20$

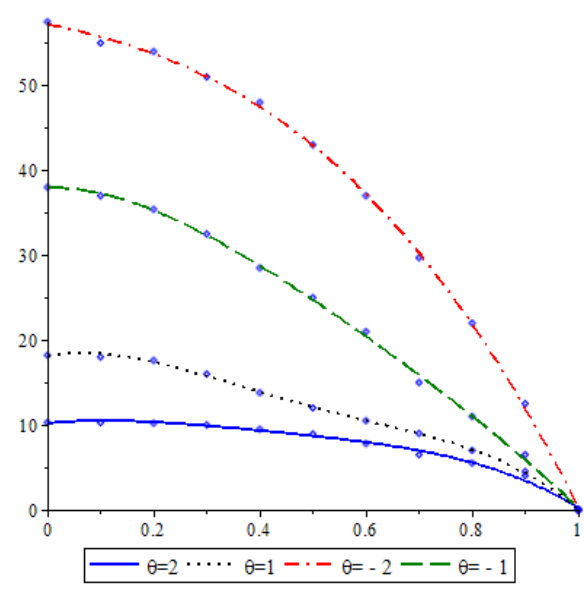

Fig. 3. The effect of angle on the heat profile for both divergent $(\theta>0)$ and convergent $(\theta<0)$ channels

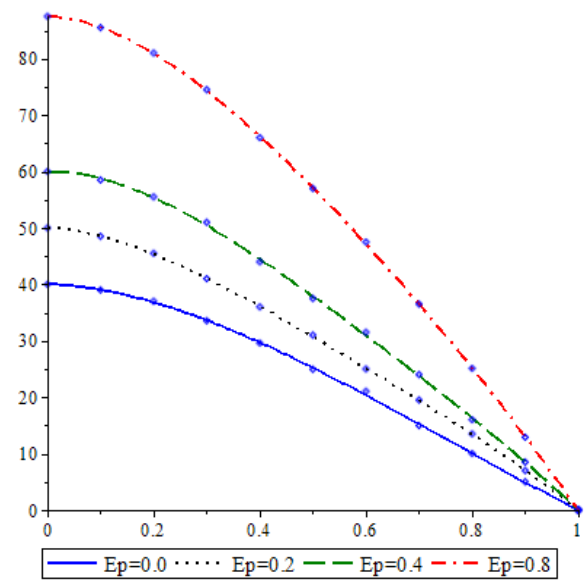

Fig. 5. The effect of $E_{p}$ on heat profile for for $\theta=2.5$ and $\mathrm{Re}=20$

From Figures 4 and 5, it can be observed that the velocity profile of Jeffery Hamel nano-fluid flow decreases as the value of $E_{p}$ increases, on the contrary the heat profile of Jeffrey Hamel nano-fluid flow also increases. 


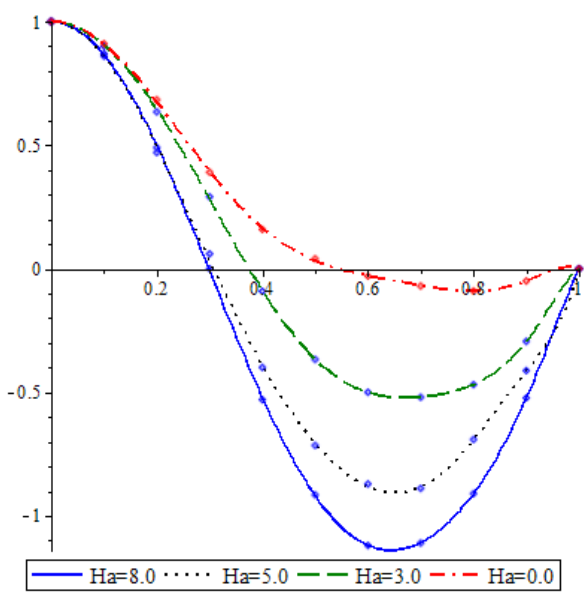

Fig. 6. The effect of Ha on velocity profile for $\theta=2.5$ and $\mathrm{Re}=20$

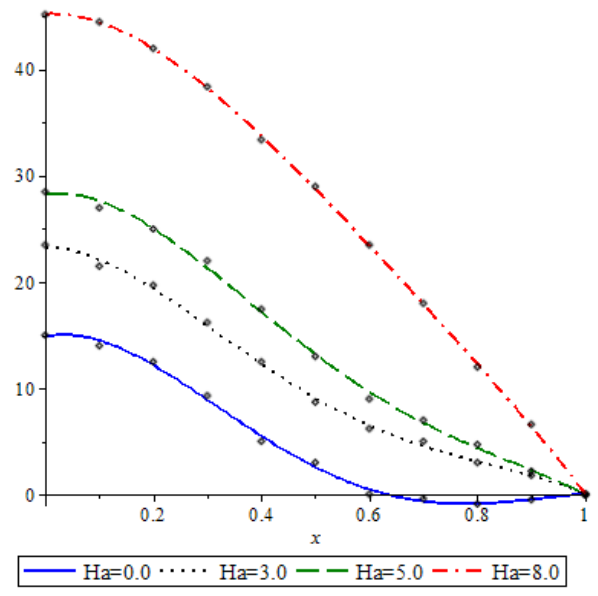

Fig. 7. The effect of Ha on heat profile for $\theta=2.5$ and $\operatorname{Re}=20$

From Figures 6 and 7, it can be seen that the velocity profile of Jeffrey Hamel nanofluid flow decreases as the value of the Ha increases, on the contrary the heat profile of Jeffrey Hamel nano-fluid flow also increases. Similarly from Figure 8, it can be deduced that the velocity profile of Jeffrey Hamel nano-fluid flow decreases with the value of Reynolds number increases.

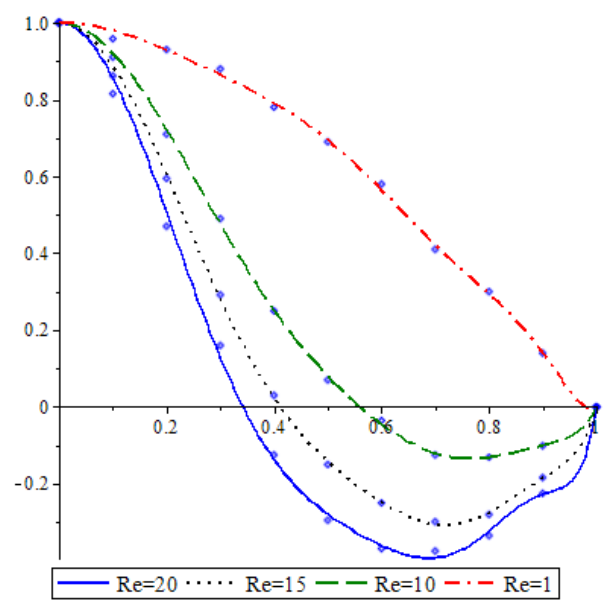

Fig. 8. The effect of Reynolds number Re on the velocity profile for $\theta=2.5$ and $E_{p}=0.2$

Similarly, from Figures 9 and 10, it can be seen that the heat profile of Jeffrey Hamel nano fluid flow increases as the value of the Eckert number and the Prandtl number increases. 


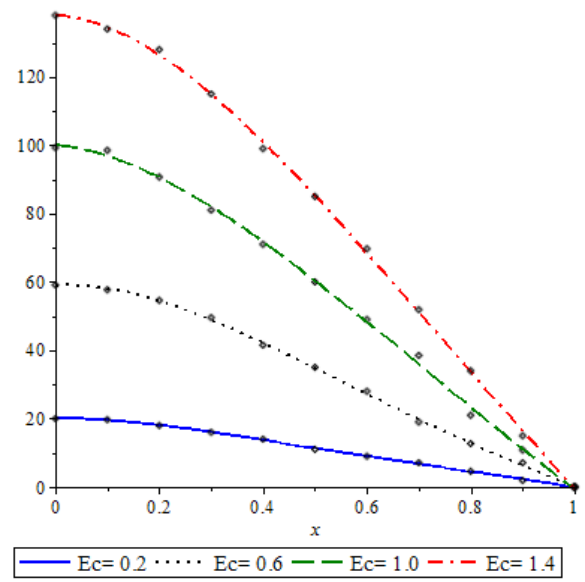

Fig. 9. The effect of Eckert number $E_{c}$ on the heat profile for $\theta=2.5$ and $\mathrm{Re}=20$

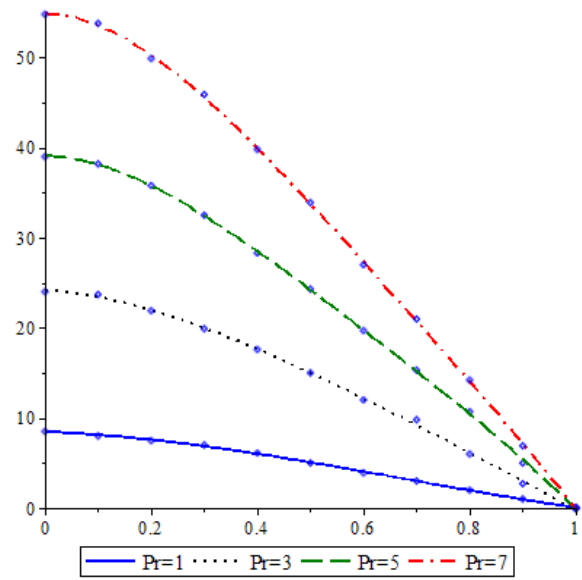

Fig. 10. The effect of Prandtl Pr on the heat profile with $\theta=2.5$ and $E_{p}=0.2$

\section{Conclusions}

In this article, the flow behaviour of the velocity profiles in MHD Jeffery-Hamel fluid flow between two unparalleled plates with heat transfer in Eyring-Powell fluid is discussed using the Differential Transform Method at different slopes fora different Eckert Number, Prandtl number, Reynolds number and Hartmann number in both convergent and divergent channels. Similarly the efficiency of the present method has been obtained by comparing the obtained results with the available numerical results. It can be concluded that the Differential Transform Method is a reliable method that provides the solution in the form of a convergent series and it can be easily handled in analysing the effect of different parameters on both velocity profiles as well as on the heat transfer in MHD Jeffery-Hamel fluid flow.

\section{References}

[1] Jeffery, G.B. (1915). The two-dimensional steady motion of a viscous fluid. Philosophical Magazine Series, 6, 29, 455-465.

[2] Hamel, G. (1917). Spiralformige bewegungen zaher flussigkeiten. Jahresbericht der Deutschen Mathematiker-Vereinigung, 25, 34-60.

[3] Akulenko, L.D., Georgevskii, D.V., \& Kumakshev, S.A. (2004). Solutions of the Jeffery-Hamel problem regularly extendable in the Reynolds number. Fluid Dynamics, 39, 1, 12-28.

[4] Makinde, O.D., \& Mhone P.Y. (2006). Hermite-Padé approximation approach to MHD Jeffery-Hamel flows. Appl. Math. Comput., 181, 966-972.

[5] Esmaili, Q., Ramiar, A., Alizadeh, E., \& Ganji, D.D. (2008). An approximation of the analytical solution of the Jeffery-Hamel flow by decomposition method. Phys. Lett., 372, 3434-3439.

[6] Rivkind, L., \& Solonnikov, V.A. (2000). Jefery Hamel asymptotics for steady state Navier Stokes flow in domains with sector-like outlets to infinity. J. Math. Fluid Mech, 324-352. 
[7] Egashira, R., Fujikawa, T., Yaguchi, H., \& Fujikawa, S. (2018). Microscopic and low Reynolds number flows between two intersecting permeable walls. Fluid Dynamics Research, 50(3), 035502 .

[8] Rana, P., Shukla, N., Gupta, Y., \& Pop, I. (2019). Analytical prediction of multiple solutions for MHD Jeffery-Hamel flow and heat transfer utilizing KKL nano-fluid model. Physics Letters A, 383(2-3), 176-185.

[9] Muhammad, U., Muhammad, H, Khan, U, Syed, T, Muhammad, A., \& Wei W. (2017). Differential transform method for unsteady nano-fluid flow and heat transfer. Alexandria Engineering Journal (in press).

[10] Zhou, J.K, \& Pukhov. (1986). Differential Transformation and Application for Electrical Circuits. Wuhan, China: Huazhong University Press.

[11] Hossein, J., Maryam, A., \& Hale T.(2010). Two dimensional differential transform method for solving non-linear partial differential equations. International Journal of Research and Reviews in Applied Sciences, 2, 1, 47-52.

[12] Patil, N., \& Khambayat, A. (2014). Differential transform method for system of linear differential equations. Research Journal of Mathematical and Statistical Sciences, 2(3), 4-6.

[13] Chen, C.K., \& Ho, S.H. (1999). Solving partial differential equations by two dimensional differential transform method. Applied Mathematics and Computation, 106, 171-179.

[14] Ayaz, F. (2004). Solutions of the systems of differential equations by differential transform method. Appl. Math. Comput., 147, 547-567.

[15] Nazari, D., \& Shahmorad, S. (2010). Application of the fractional differential transform method to fractional-order integro-differential equations with nonlocal boundary conditions. J. Comput. Appl. Math., 234, 883-891.

[16] Farshid, M., \& Mohammad, K.Y. (2016). A novel computing three dimensional differential transform method for solving fuzzy partial differential equations. Ain Shams Engineering, 7, 695-708.

[17] Farshid, M. (2011). Differential transform method for solving linear and nonlinear systems of ordinary differential equations. Applied Mathematical Sciences, 5(70), 3465-3472.

[18] Patel, H.S., \& Meher, R. (2016). Analytical investigation of Jeffery-Hemal flow by modified adomian decomposition method, Ain Shams Engineering Journal.

[19] Patel, N.D., \& Meher, R. (2016). Differential transformation method for solving KolmogrovePetrovskii-Piskunov equation and porous medium equation. Mathematical Sciences, IMRF Journals, 5, 1, 47-51.

[20] Patel, N.D., Meher R. (2017). Differential transform method for solving for fingero-imbibition phenomena arising in double phase flow through homogeneous porous media. Mathematical Sciences, IMRF Journals, 6, 1, 1-5.

[21] Rana, P., Shukla, N., Gupta, Y., \& Pop, I. (2019). Homotopy analysis method for predicting multiple solutions in the channel flow with stability analysis. Communications in Nonlinear Science and Numerical Simulation, 66, 183-193. 